\title{
A PARADIGM FOR DESIGN AND MANUFACTURING BASED ON ADDITIVE MANUFACTURING (3DP) ONLINE PLATFORM
}

\section{KIANOUSH HAGHSEFAT \& TINGTING LIU}

Department of Mechanical Engineering, Nanjing University of Science and Technology, Nanjing, China

\begin{abstract}
Cloud manufacturing is a modern paradigm of intelligent, effective, and service oriented production that has been proposed in recent years. This paper applies the $3 D$ printing industry to the cloud manufacturing model which by combining these two new eras of manufacturing a new manufacturing system namely collaborative design and manufacturing paradigm in the intelligent platform of additive manufacturing is born.

According to this research, the system design and key technologies of the additive manufacturing intelligent cloud platform are systematically analyzed on the basis of the cloud manufacturing concept, a system architecture has been proposed and Using PHP Language and MySQL Server a WordPress web server which represents as prototype platform has been developed and finally based on the current technological issues advantages and disadvantages of this new era of manufacturing is analyzed.

KEYWORDS: 3D Printing, Cloud Manufacturing, AM Online Platform, Collaborative Design and manufacturing, Modeling Method of Resources and Tasks
\end{abstract}

Received: Nov 27, 2020; Accepted: Dec 18, 2020; Published: Dec 28, 2020; Paper Id.: IJMPERDFEB20211

\section{INTRODUCTION}

In the 21 st century when the manufacturing industry is becoming more and more international, personalized service and market response speed have become the key for most companies to survive. As a result, a large number of advanced manufacturing concepts and manufacturing technologies have emerged, and 3D printing technology is one of them. 3D printing technology is a major breakthrough in the field of manufacturing technology [1]. It can quickly and accurately convert design model files into actual parts or product prototypes that meet certain functional requirements, shortening the development cycle of new products and improving market response. Speed is of great help [2-3]. Although 3D printing technology is developing rapidly, the current growth rate of the 3D printing consumer market is relatively slow.

The main reasons are the following:

- The lack of active cooperation and information exchange in the $3 \mathrm{D}$ printing industry makes it difficult to form a complete 3D printing service industry Chain [4];

- Most 3D printing companies, research institutes and institutions have not built a good external service information platform, resulting in a high idle rate of resources;

- It is difficult for users to obtain suitable 3D printing services. On the one hand, most users do not have a high degree of trust in small and medium-sized 3D printing network platforms. On the other hand, users who want to obtain suitable $3 \mathrm{D}$ printing services often need to collect a lot of information to compare and 
weigh, which to a certain extent causes users to find services. The rent cost is higher.

Cloud manufacturing is a new model of service-oriented, high-efficiency, low-consumption, and knowledgebased networked intelligent manufacturing[5-6]. It virtualizes and serves various manufacturing resources and manufacturing capabilities, and performs unified and centralized intelligence. To achieve the purpose of providing users with on-demand manufacturing[7-8]. Yuan Chunmei et al. [9] applied the cloud manufacturing model to the field of EDM to strengthen the information cooperation between EDM enterprises and users. Gu Xinjian et al. [10] applied the cloud manufacturing model to the field of mold manufacturing to help mold companies expand to the service industry and to the upstream and downstream industries.

This paper applies the cloud manufacturing model to the field of Additive Manufacturing also known as 3D printing, in order to build a 3D printing cloud(online) platform as shown in Figure 1 with free access and efficient scheduling of resources, and studies its key technologies such as systems architecture. The 3D printing cloud platform integrates scattered 3D printing resources and capabilities to form a huge virtual resource pool, combined with the platform's efficient resource management and scheduling mechanism, to maximize the utilization of idle resources and minimize the rent-seeking cost of user services.

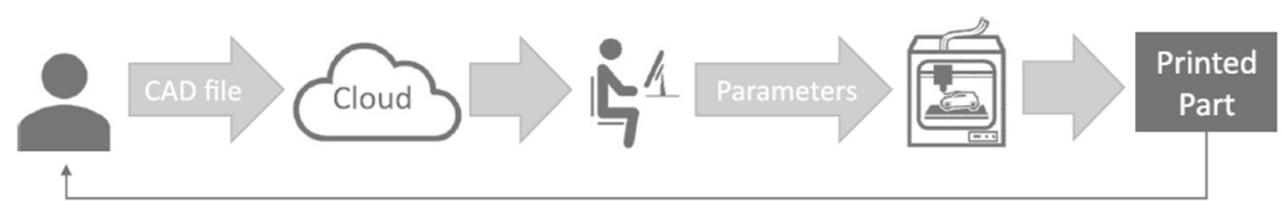

Figure 1 : Basic schematic view of 3D printing cloud platform over view

\section{THE ARCHITECTURE OF THE AM CLOUD PLATFORM}

With reference to the multi-layer system architecture of cloud manufacturing [11], the system structure of service platform for 3D printing products is proposed. The cloud platform's architecture has a total of eight layers, which are the basic technology support layer, 3D printing resource layer, virtual resource layer, service resource layer, tool layer, application interface layer, access layer, and user layer. The architecture diagram of the cloud service platform is shown in Figure 2. 


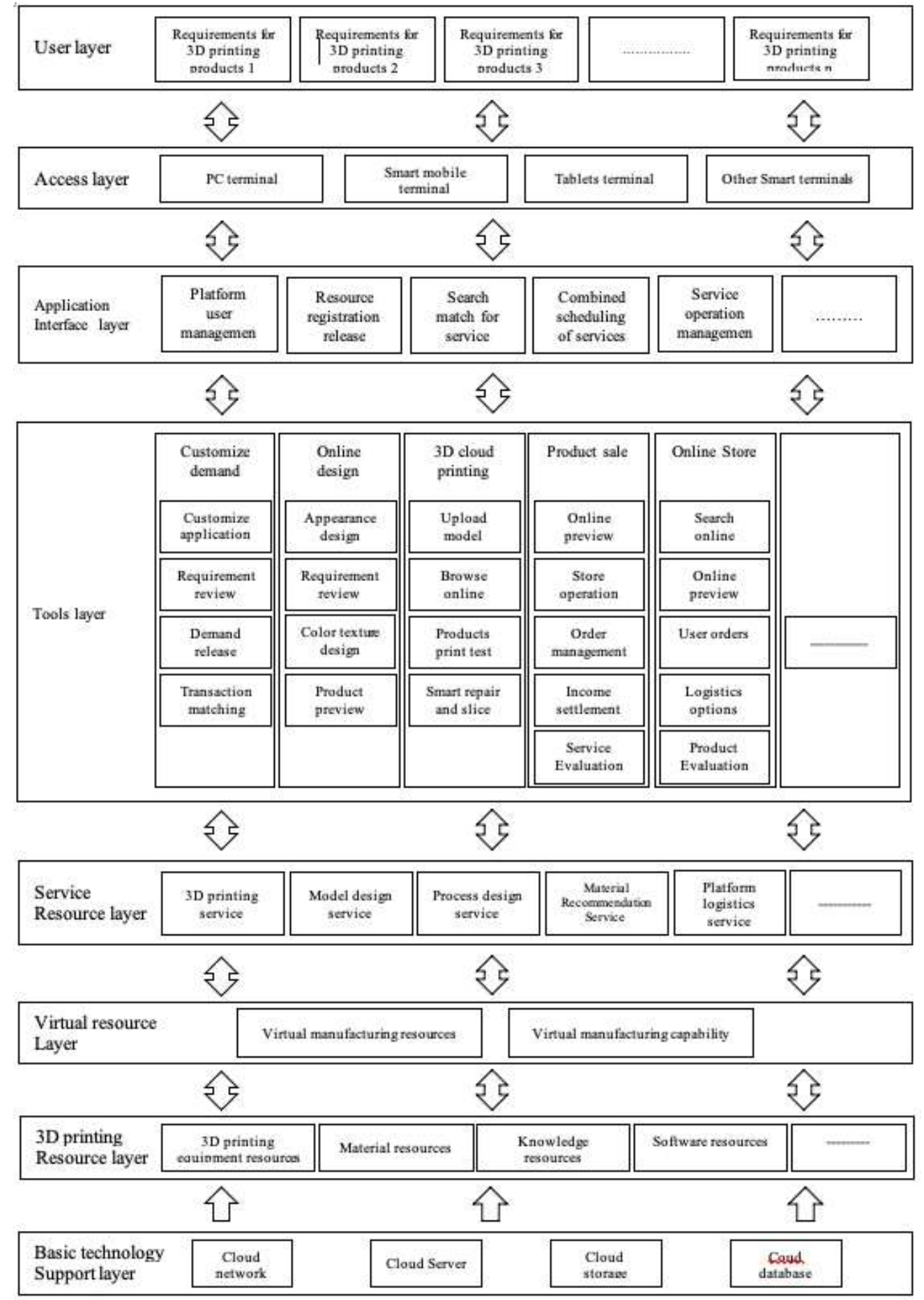

Figure 2 : AM 3D printing cloud service platform architecture diagram

- Basic Technology Support Layer: The basic technology support layer is the basic layer of the cloud service platform [12]. Cloud service platforms need to use infrastructure as a service The purpose of the management model is to provide a stable and smooth technical support for the operation of the cloud manufacturing service platform. Which mainly categorizes in three different sections as , 1) cloud network ,2) cloud server and 3)cloud storage.

- 3D printing resource layer : The 3D printing resource layer is the physical foundation of the entire cloud platform, 
providing support for the operation of various services in the cloud platform. Generally speaking, 3D printing resources can be divided into two categories: 3D printing hard and soft manufacturing resources. Among them, 3D printing hard manufacturing resources mainly include various types of equipment resources (such as printing equipment, scanning equipment, testing equipment, etc.), material resources (such as 3D printing materials, parts, etc.); 3D printing soft manufacturing resources mainly include various types of software resources (Such as 3D drawing software, service billing software, stress analysis software, etc.), human resources (such as model file designers, industry experts, managers, etc.), knowledge resources (such as 3D printing examples, industry standards, technical references, etc.).

- Virtual resource layer : The main function of the virtual resource layer is to abstract and simplify the 3D printing resources connected to the cloud service platform [13]. First, use the virtualization technology in the cloud service platform to abstractly describe the attribute information of various 3D printing physical resources and describe it as virtual resource information to form virtual data resources; secondly, the virtual data resources are encapsulated and released to the cloud service platform The resource service center module in, forms a virtual resource cloud pool, and finally users can choose various printing resources they need from the cloud.

- Service resource layer: This layer is the core of the entire cloud platform, which mainly implements serviceoriented packaging and management of virtual resources and manufacturing capabilities. Using service-based packaging technology to encapsulate virtual resources and manufacturing capabilities as services, to shield the heterogeneity and complexity of the resources themselves, to present a unified service interface to the outside, so as to achieve convenient invocation in the cloud platform environment. Cloud service management mainly implements management functions such as storage, update, and maintenance of cloud services.

- Tools layer: The main function of the tool layer is that the cloud service platform provides users with good human-computer interaction application services and realizes the ease of operation and functional convenience of the cloud service platform. The tool layer is provided by the cloud service platform operator, which mainly includes modules such as demand release, online design, cloud 3D printing, product sales, and shopping cart.

- Requirements release module: The main function of the this section is to apply, review, and release specific user requirements And transactions, and then connect user needs to the cloud service platform for users to use online design module :The main function of this section is online design module is the appearance design, material selection, Color texture design and effect preview of 3D printed products.

- 3D cloud printing module : The main function of the $3 \mathrm{D}$ cloud printing module is to personalize the user to upload the 3D model of the product to the cloud service platform, browse online after uploading, and then perform product simulation printing detection on the product to be printed, and finally use the software to determine the user's personality Intelligently repair the 3D model diagram of customized products.

- Product sales module the main functions of the product sales module includes online preview, store operation, product order management, revenue settlement and services evaluation etc.

- Shopping cart module: the shopping mall shopping module includes functions such as online search, online preview, user orders, logistics selection and product evaluation, etc.

- Application interface layer : This layer provides various application interfaces that are easy for users to access, so that users can freely and transparently call various cloud services. The application interface provided by the 
platform mainly includes platform user management, resource registration and release, service search and choosing, service combination scheduling, and service operation management.

- Access layer: The access layer is the terminal access layer where users of the product need to interact with the cloud service platform. The cloud service platform supports a variety of terminal types for access, including PC terminals, mobile smart terminals, and dedicated terminals, to achieve dynamic responsiveness compatibility for different terminals .

- User layer: This layer is a bridge between users and the cloud platform. Users can access and invoke cloud services through universal terminal interaction devices such as PC terminals, mobile tablets, and mobile terminal phones.

The 3D printing cloud platform can dynamically combine platform resources to form cloud services according to user needs. Users can obtain high-quality and low-cost services on demand, and monitor the resources being served through the support of Internet of Things technology.

\section{AM3D PRODUCT DESIGN AND MANUFACTURING THROUGH CLOUD SERVICE PLATFORM PROTOTYPE DESIGN}

\section{Development Tools for AM Cloud Service Platforms}

The program of the system server is quite complicated, but the existence of software's simplifies the program of the system server which needs to be handled by the programmer. In this case, the programmer only needs to be responsible for the logic part of the software program.

The available software development tools are as follows:

- (1) Web server : The exchange of data information between the Web server and the browser requires data connection, server request, and browser response. The Apache server is characterized by good versatility and can quickly and efficiently complete the interaction between the server and the browser. Therefore, it is the best choice for Web servers.

- (2) Programming language : To have a good operation of the system, programming language must be used as the foundation. The PHP language is a universal scripting language. The programming language is relatively simple and widely used. The most important thing is that it is an open source programming language. There are many open source codes for programmers to refer to. It is a great tool to use it as the programming language of the cloud service platform.

- (3) Database : The database is the basis for the storage and use of all databases on the cloud service platform, and has the ability to organize, store and manage data. The cloud manufacturing service platform has a large number of 3D printing manufacturing resources and manufacturing capabilities data information, so a database with good performance and easy to use and operate is more needed. MySQL database has the characteristics of relatively small system memory, faster reading and running speed, and relatively low cost. More importantly, it is open source code for use and has good interaction performance with the PHP programming language through WordPress. Therefore, MySQL database is selected as the database of the online service platform. According to Figure 3-6 respectively (user information model table, three dimensional model file,3d printing equipment model file and $3 \mathrm{~d}$ printing order model file) is proposed . 


\begin{tabular}{|c|c|c|c|c|}
\hline Field name & type & length & $\begin{array}{c}\text { Allowed to be empty } \\
\text { (YN) }\end{array}$ & Primary key \\
\hline id & vatchar & 30 & $\mathrm{~N}$ & $\sqrt{ }$ \\
\hline username & varehar & 30 & $\mathrm{~N}$ & \\
\hline identity & char & 1 & $\mathrm{~N}$ & \\
\hline password & varchar & 30 & $\mathbf{N}$ & \\
\hline phone & varchar & 20 & $\mathrm{~N}$ & \\
\hline address & varchar & 50 & $\mathbf{Y}$ & \\
\hline email & varehar & 30 & $\mathbf{Y}$ & \\
\hline introduction & varchar & 100 & $\mathbf{Y}$ & \\
\hline Field name & type & length & $\begin{array}{c}\text { Allowed to be empty } \\
\text { (Y/N) }\end{array}$ & Primary key \\
\hline id & varchar & 30 & $\mathbf{N}$ & $\sqrt{ }$ \\
\hline printemame & varchar & 30 & $\mathbf{N}$ & \\
\hline ownerid & varchar & 30 & $\mathrm{~N}$ & \\
\hline category & char & 1 & $\mathbf{Y}$ & \\
\hline presion & float & $(3,2)$ & $\mathbf{Y}$ & \\
\hline printerablematerial & varchar & 50 & $\mathbf{N}$ & \\
\hline printerablesize & varchar & 20 & $\mathbf{N}$ & \\
\hline globalqos & float & $(3,2)$ & $\mathbf{N}$ & \\
\hline introduction & varchar & 100 & $\mathbf{Y}$ & \\
\hline
\end{tabular}

\begin{tabular}{|c|c|c|c|c|}
\hline Field name & type & length & \begin{tabular}{|c|} 
Allowed to be empty \\
(YN)
\end{tabular} & Primary key \\
\hline id & varchar & 30 & $\mathrm{~N}$ & $\sqrt{ }$ \\
\hline filename & varchar & 30 & $\mathrm{~N}$ & \\
\hline ownerid & varchar & 30 & $\mathrm{~N}$ & \\
\hline filepath| & varchar & 30 & $\mathbf{N}$ & \\
\hline category & char & 1 & $\mathbf{Y}$ & \\
\hline sales & varchar & 30 & $\mathbf{N}$ & \\
\hline price & foat & $(8,2)$ & $\mathbf{N}$ & \\
\hline quality & noat & $(3,2)$ & $\mathrm{N}$ & \\
\hline introduction & varchar & 100 & $\mathbf{Y}$ & \\
\hline Field name & type & length & $\begin{array}{c}\text { Allowed to be empty } \\
\text { (YN) }\end{array}$ & Primary key \\
\hline id & varchar & 30 & $\mathrm{~N}$ & $\sqrt{ }$ \\
\hline fid & varchar & 30 & $\mathbf{N}$ & \\
\hline buyerid & varchar & 30 & $\mathrm{~N}$ & \\
\hline providerid & varchar & 30 & $\mathrm{~N}$ & \\
\hline issuccesss & char & 1 & $\mathrm{~N}$ & \\
\hline time & varchar & 20 & $\mathrm{~N}$ & \\
\hline quality & float & $(3,2)$ & $\mathrm{N}$ & \\
\hline category & char & 1 & $\mathrm{~N}$ & \\
\hline
\end{tabular}

Figure 3-6: User functional module through the online 3D platform

\section{Main Functional Modules of AM Cloud Platform}

According to the different functions required by different users in the 3D printing cloud platform, the main functional modules of the platform are designed as shown in Figure 7 Users can obtain the corresponding function permissions after registering and logging in. For ordinary users, the main functions provided include personal information management, service choosing, order management, refund rights protection, etc. Among them, Design to production is the core function provided by the platform, including material recommendation, design request, printing service recommendation and other functions. Users can Use it freely according to their own needs. For designer users, the main functions provided include personal information management, model file management, request management, order management, refund management, etc. The model file management mainly includes file upload, deletion, and file information maintenance. For users of printing service providers, the main functions provided include personal information management, printing device management, order management, refund management, etc. The printing device management mainly includes functions such as device virtualization access, device information maintenance, and device deletion. 


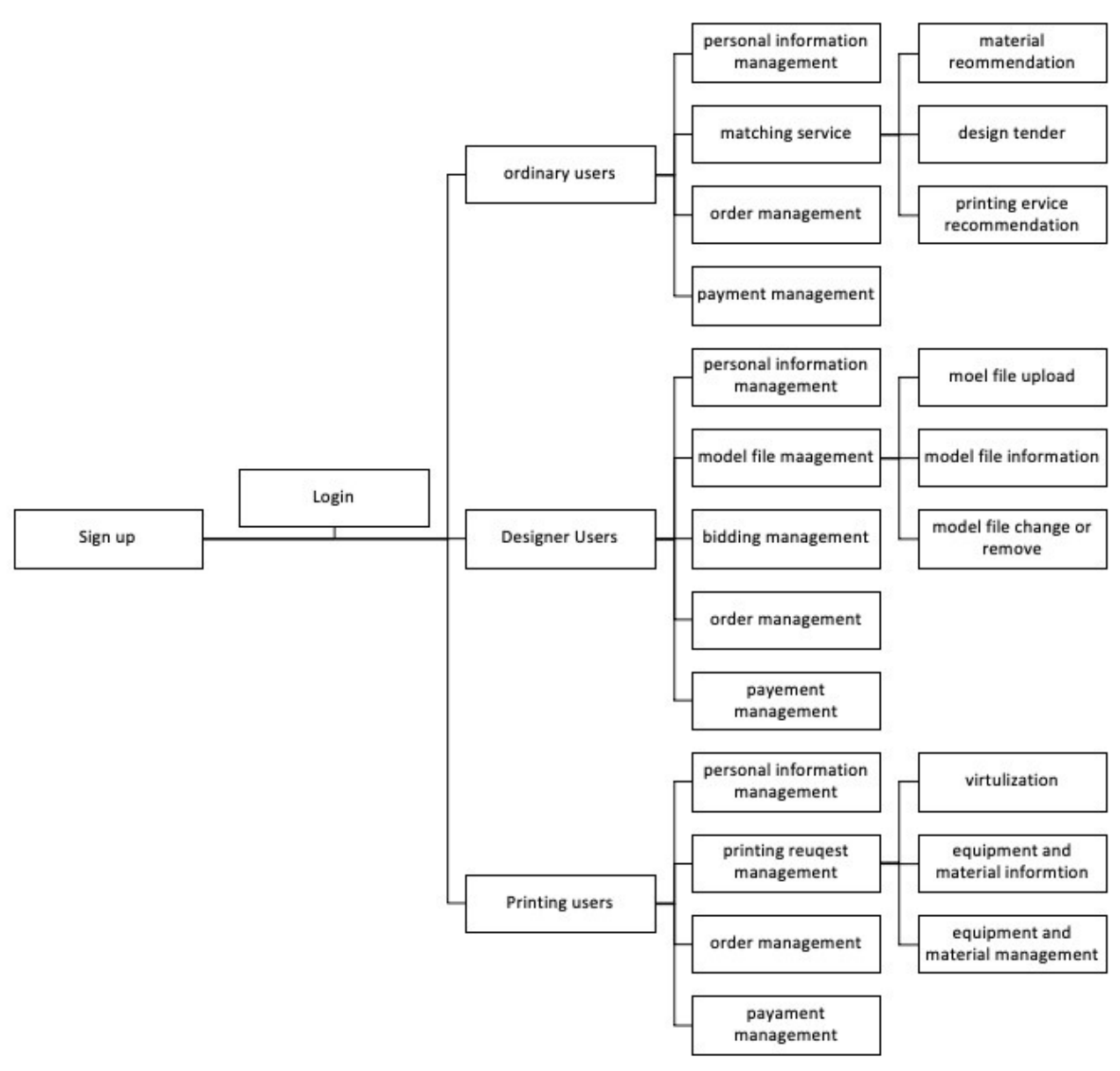

Figure 7: User functional module through the online 3D platform

\section{D Printing Cloud Platform Navigation (Main panel) Interface}

The cloud service platform for 3D printing products is the design and optimization of the front-end main page based on the overall construction design of the Nanjing University of Science and Technology Cloud AM Service Platform I 3D Printing Products Cloud Service which applied through https://arcmfg-njust.ir/ which is the main page of the Additive cloud design and manufacturing service platform, it consists of the main module as navigation panel and multiple submodule pages related to operating (User) interface panels.

\section{D Printing Cloud Platform Operating (User) Interface}

Users can obtain the corresponding function permissions after registering and logging in , the main functions provided include personal information management, model file management, request management, order management, refund management, etc. The model file management mainly includes file upload, deletion, and file information maintenance. The 3D printing cloud platform has a massive 3D model file library and designers, and users can actively search for suitable service resources. In addition, users can also upload their design and printing requirements, allowing many designers on the platform to actively provide customers with customized services.. 
Once the user has determined the 3D model files required for printing, based on their need of different materials , coating, they can submit a print job request to their desired printing service and at last choosing the desired quantity After the input of various parameters is completed and the user submits a model request, the cloud platform system will show the service according to the users requests mentioned in the above chapter, and finally move to the purchase bucket. As shown in Figure 8 a full cycle from registration and membership upgrading, uploading design model, Request matching and production is shown.

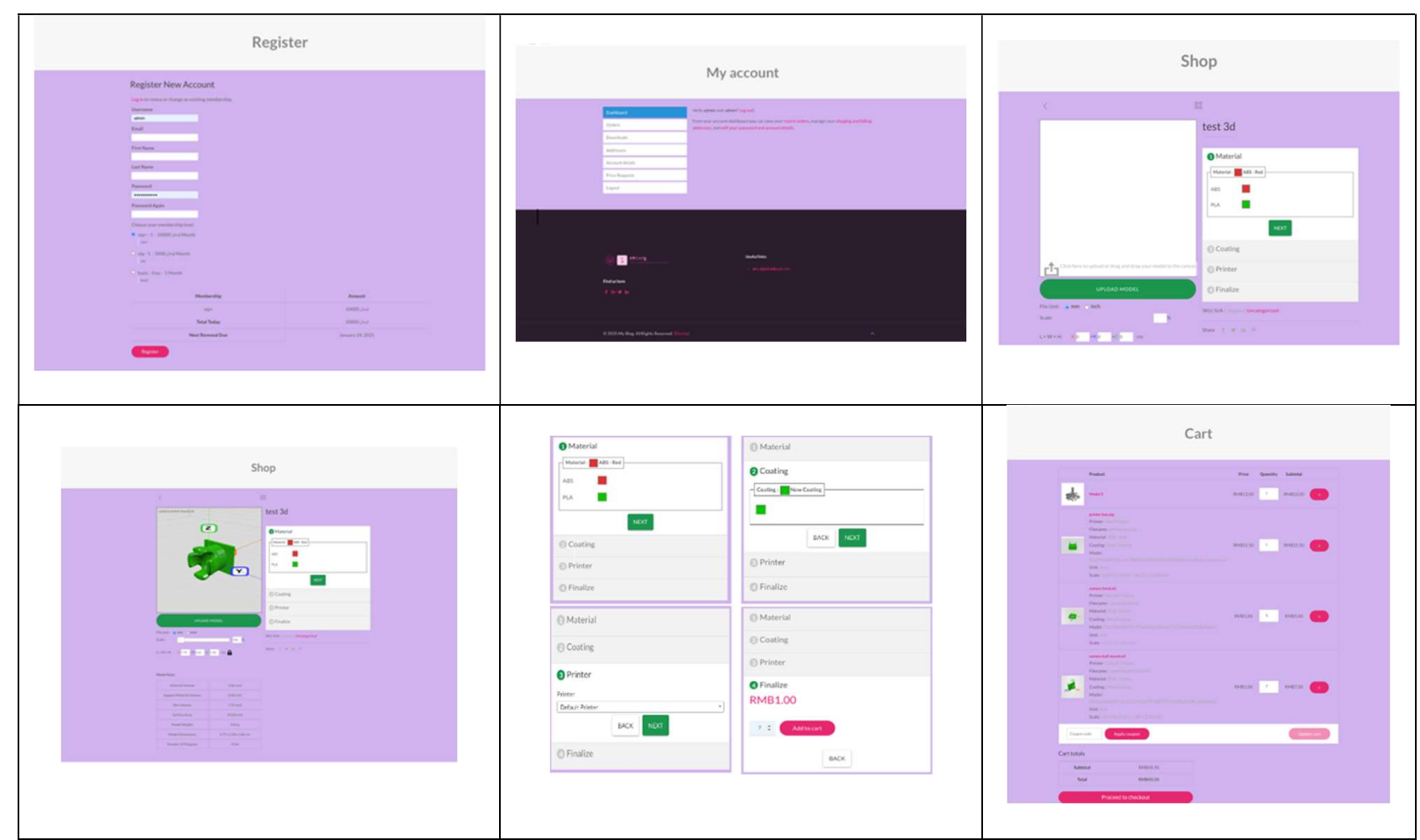

Figure 8 : Full cycle of AM cloud platform based on design and manufacturing

\section{CONCLUSIONS}

The aim of this research was to gain basic understanding and hands on experience in design to manufacturing through AM online Platform, In view of the current difficulties in the development of the worldwide 3D printing industries, such as insufficient resource integration capabilities and insufficient service models, this article applies the design and manufacturing of $3 \mathrm{~d}$ printing online platform to the $3 \mathrm{D}$ printing industry.

The construction of a 3D printing cloud platform involves many information technologies, which is an extremely complex issue. Due to the limited resources there are still some shortcomings in the research, which need further study.

- (1) Security issues of the 3D printing cloud platform. The security of resource information under the cloud platform is the basic issue that the cloud platform needs to solve and the key to the long-term stable operation of the cloud platform.

- (2) The cost and time acquisition of 3D printing services. The cost and time can be obtained in real time in this article, but it has not been studied in depth. The feedback rate of 3D printing service cost and time will have a great impact on cloud platform user experience. It is necessary to standardize industry standards to reduce the difficulty of real-time acquisition. This is a technical problem to be solved in the future. 
- (3) The issue of rational use of knowledge resources. The cloud platform can accommodate a large amount of knowledge and information. How to use this knowledge and information to promote the standardization of the 3D printing industry and expand the interests of both supply and demand is the focus of future research.

- (4) The practical application of 3D printing cloud platform. In the preliminary form of the prototype system knowledge cloud platform developed in this paper, there are still many factors that have not been considered properly, and the cloud platform needs to be further expanded to make the cloud platform adapt to the actual business environment.

Following Figure 9 shows the advantages and disadvantages of Additive manufacturing online platform which is analyzed due to comparison of the findings of this study and a comparison to similar researches.

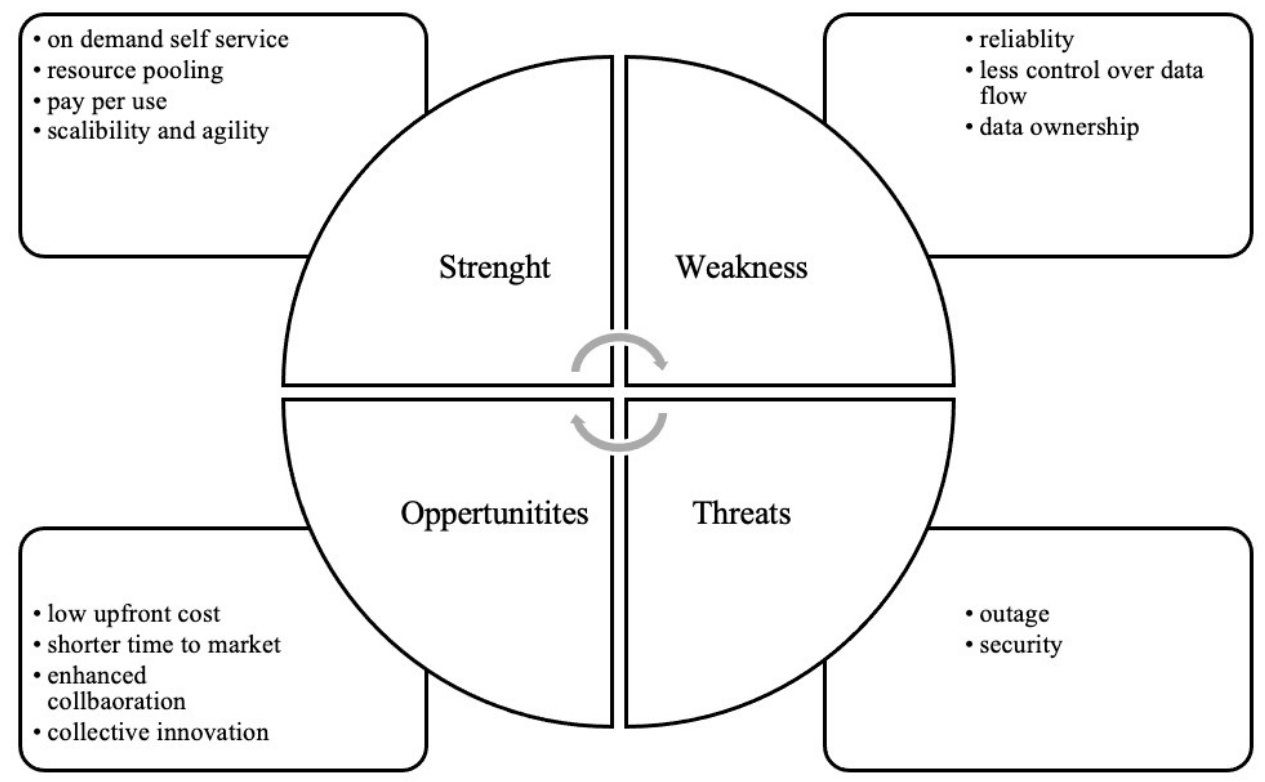

Figure 9 : SWOT Analysis of AM cloud Platform

\section{REFERENCES}

1. GUNTHER D, HEYMEL B, GUNTHER J F, et al. Continuous 3D-printing for additive manufacturing[ J]. Rapid Prototyping Journal, 2014, 20(4): 320-327.

2. Wang Guangchun, Zhao Guoqun. Rapid prototyping and rapid mold manufacturing technology and its application [M]. Beijing: Machinery Industry Press, 2009.

3. Huang Shuhuai, Xiao Yuejia, Mo Jianhua, etc. The prospect of rapid prototyping technology [J]. China Mechanical Engineering, 2000, 28(11): 195-200.

4. Ma Min, Wang Ling. Can 3D printing change the manufacturing industry? An interview with Lu Bingheng, an academician of the Chinese Academy of Engineering [J]. High Technology and Industrialization, 2013, 19(4): 38-43.

5. TAO F,HU Y F,ZHOU Z D. Study on manufacturing grid \& its resource service optimal-selection system [J ]. International Journal of Advanced Manufacturing Technology, 2008, 37 (9 / 10): 1022-1041.

6. Li Bohu, Zhang Lin, Ren Lei. Discuss Cloud Manufacturing Again [J]. Computer Integrated Manufacturing System, 2011, 17(3): 449-457. 
7. HUANG B, LI C, TAO F. A chaos control optimal algorithm for QoS-based service composition selection in cloud manufacturing system [J]. Enterprise Information Systems, 2014, 8 (4): 445-463.

8. Li Bohu, Zhang Lin, Chai Xudong, et al. Cloud manufacturing [M]. Beijing: Tsinghua University Press, 2015. [9] Yuan Chunmei, Yang Xiaodong, Chen Tao. Intelligent cloud platform for electrical processing technology based on cloud manufacturing [J]. Electrical Machining and Mould, 2016, 14(2): 11-14.

9. Yuan Chunmei, Yang Xiaodong, Chen Tao. Intelligent cloud platform for electrical processing technology based on cloud manufacturing [J]. Electrical Machining and Mould, 2016, 14(2): 11-14.

10. Gu Xinjian, Huang Shenquan, Chen Jixi, et al. Demand-driven cloud manufacturing service platform in the mold industry [J]. Computer Integrated Manufacturing System, 2012, 18(7): 449-457.

11. Li Bohu,Zhang Lin,Wang Shilong, et al. Cloud Manufacturing-a new service-oriented networked manufacturing model[J].Computer Integrated Manufacturing System,2010,16(01):1-7+16.

12. Liu Yonghui, Yin Zuozhong, Huang Shuangxi, et al. Research on the architecture of $3 D$ printing cloud service platform[J].Manufacturing Automation, 2017,39(06):145-149+156

13. Chen Tao. Research on EDM Cloud Platform Based on Cloud Manufacturing[D]. Harbin: Harbin Institute of Technology, 2013. 